\title{
Prognostic impact of pre-existing interstitial lung disease in non-HIV patients with Pneumocystis pneumonia
}

\author{
Shohei Hamada', Hidenori Ichiyasu', Megumi Inaba², Hiroshi Takahashi ${ }^{1}$, \\ Tomoki Sadamatsu ${ }^{3}$, Kimitaka Akaike ${ }^{1}$, Aiko Masunaga ${ }^{1}$, Yasumasa Tashiro ${ }^{3}$, \\ Naomi Hirata ${ }^{2}$, Takeshi Yoshinaga ${ }^{2}$ and Takuro Sakagami ${ }^{1}$
}

Affiliations: ${ }^{1}$ Dept of Respiratory Medicine, Kumamoto University Hospital, Faculty of Life Sciences, Kumamoto University, Kumamoto, Japan. ${ }^{2}$ Division of Respiratory Medicine, Kumamoto Chuo Hospital, Kumamoto, Japan. ${ }^{3}$ Dept of Respiratory Medicine, Minamata City General Hospital and Medical Center, Minamata, Japan.

Correspondence: Hidenori Ichiyasu, Dept of Respiratory Medicine, Faculty of Life Sciences, Kumamoto University, 1-1-1 Honjo, Chuo-ku, 860-8556, Kumamoto, Japan. E-mail: ichiyasuQkumamoto-u.ac.jp

\section{ABSTRACT}

Background: The increasing incidence of life-threatening Pneumocystis pneumonia (PCP) in non-HIV immunocompromised patients is a global concern. Yet, no reports have examined the prognostic significance of pre-existing interstitial lung disease (ILD) in non-HIV PCP.

Methods: We retrospectively reviewed the medical records of non-HIV PCP patients with (ILD group) or without (non-ILD group) pre-existing ILD. The clinical features and outcomes of the ILD group were compared with those of the non-ILD group. Cox regression models were constructed to identify prognostic factors.

Results: 74 patients were enrolled in this study. The 90-day mortality was significantly higher in the ILD group than in the non-ILD group $(62.5 \%$ versus $19.0 \%, \mathrm{p}<0.001)$. In the ILD group, patients with a higher percentage of bronchoalveolar lavage fluid neutrophils had worse outcomes compared to those having a lower percentage $(p=0.026)$. Multivariate analyses revealed that pre-existing ILD $(p=0.002)$ and low levels of serum albumin $(p=0.009)$ were independent risk factors for 90 -day mortality. Serum levels of $\beta$-Dglucan were significantly reduced after treatment of PCP in both groups, whereas levels of Krebs von den Lungen-6 (KL-6) significantly increased in the ILD group. In the ILD group, the 90-day mortality of patients with increasing KL-6 levels after treatment was significantly higher than those with decreasing levels $(78.9 \%$ versus $0 \%, \mathrm{p}=0.019)$.

Conclusion: In non-HIV PCP patients, pre-existing ILD is associated with a poorer prognosis. Prophylaxis for PCP is needed in patients with pre-existing ILD under immunosuppression.

@ERSpublications

Pre-existing interstitial lung disease (ILD) is an independent prognostic risk factor for nonHIV Pneumocystis pneumonia (PCP). Prophylaxis for PCP is needed in patients with preexisting ILD under immunosuppression. http://bit.ly/37BGZuK

Cite this article as: Hamada S, Ichiyasu $\mathrm{H}$, Inaba $\mathrm{M}$, et al. Prognostic impact of pre-existing interstitial lung disease in non-HIV patients with Pneumocystis pneumonia. ERJ Open Res 2020; 6: 00306-2019 [https://doi.org/10.1183/23120541.00306-2019].

This article has supplementary material available from openres.ersjournals.com

Received: 6 Nov 2019 | Accepted after revision: 11 Feb 2020

Copyright $\odot$ ERS 2020. This article is open access and distributed under the terms of the Creative Commons Attribution Non-Commercial Licence 4.0. 


\section{Introduction}

In recent years, the incidence of Pneumocystis jirovecii pneumonia (PCP) in AIDS patients has decreased due to the use of chemoprophylaxis with highly active antiretroviral therapy $[1,2]$. However, PCP in non-HIV immunocompromised patients is increasing because immunosuppressive therapies and chemotherapeutic agents have been frequently used in a broad range of diseases, including malignancies and autoimmune disorders [3]. The mortality rate of PCP in non-HIV patients ranges from $19.6 \%$ to $52.9 \%$ [4-6], which is significantly higher than in HIV-infected patients [1, 6, 7]. Several risk factors, including female sex, co-infections, solid tumours, severe respiratory failure, high lactate dehydrogenase (LDH) levels, delay of initial treatment, low serum albumin and chronic pulmonary diseases, have been reported to be associated with a poor prognosis in non-HIV patients with PCP [5-8]. Few studies, however, have investigated the potential association with pre-existing interstitial lung disease (ILD), which often coexists with underlying diseases such as connective tissue disease (CTD) and lung cancer. Although a high incidence of PCP has been observed in patients with idiopathic pulmonary fibrosis (IPF) receiving corticosteroid therapy [9], it is unclear whether pre-existing ILD is associated with the prognosis of non-HIV PCP patients.

Several studies have described the association of severe infection with a poorer prognosis in patients with ILD [10-12]. Moua et al. [11] reported an in-hospital mortality rate of $49 \%$ for fibrotic ILD with acute respiratory failure (ARF) due to various causes including infection. In another study, in-hospital mortality of ILD patients with ARF was 66\% [12]. Thus, the mortality of ILD with ARF was relatively high compared with acute respiratory distress syndrome (ARDS) $[13,14]$. However, the impact of infection caused by specific pathogens like $P$. jirovecii on pre-existing ILD remains unclear.

Therefore, we hypothesised that pre-existing ILD would be a risk factor associated with high mortality in non-HIV patients with PCP, and we conducted a multicentre retrospective study to elucidate clinical features and outcomes of this population.

\section{Methods}

\section{Patient selection}

We retrospectively reviewed medical records of patients who were diagnosed with PCP, with or without pre-existing ILD, who were treated at Kumamoto University Hospital (Kumamoto, Japan) and two affiliated hospitals between December 2008 and December 2018. All patients who were admitted with a diagnosis of PCP were screened for eligibility. Inclusion criteria comprised: 1) acute symptoms including fever, cough, sputum and dyspnoea; 2) newly developed high-resolution computed tomography (HRCT) findings compatible with PCP [15] after developing symptoms; and 3) positive microbiological tests for P. jirovecii confirmed by PCR or Gomori methenamine silver (GMS) stain of samples from bronchoalveolar lavage fluid (BALF) or sputum. Patients with HIV infection or patients whose levels of $\beta$-D-glucan in the observational period were $<11 \mathrm{pg} \cdot \mathrm{mL}^{-1}$, which was the cut-off value used by the WAKO assay, were excluded. Enrolled PCP patients were subdivided into two groups (ILD group and non-ILD group) according to the presence or absence of pre-existing ILD. HRCT findings of PCP and pre-existing ILD were reviewed by two pulmonologists (S. Hamada and H. Ichiyasu) who were blinded to the patient information. The study protocol was approved by the institutional review board of each participating institution (Kumamoto University Hospital, approval number 1694; Kumamoto Chuo Hospital, approval number 6503; Minamata City General Hospital and Medical Center, approval number 1901).

\section{Definition of pre-existing ILD}

Pre-existing ILD was defined according to clinical features and HRCT findings (i.e. pre-existing ground-glass opacity (GGO), consolidation and reticulation shadow in both lung fields). Patients with idiopathic interstitial pneumonias (IIPs), including IPF, were diagnosed according to the international consensus classification of the American Thoracic Society/European Respiratory Society [16]. CTD-ILD, chronic hypersensitivity pneumonia (CHP), chronic eosinophilic pneumonia (CEP), asbestosis and radiation pneumonitis were diagnosed in accordance with established criteria [17-21]. HRCT findings of pre-existing ILD were classified using a previously published clinical practice guideline [22] into two groups: usual interstitial pneumonia (UIP) and non-UIP patterns.

\section{Analysis of BALF}

Bronchoalveolar lavage (BAL) was performed through a fibreoptic bronchoscope using standard techniques. The BALF was pooled and centrifuged at $200 \mathrm{~g}$ for $5 \mathrm{~min}$ at $4^{\circ} \mathrm{C}$. Cells from BALF samples were resuspended in $10 \mathrm{~mL}$ normal saline. The cell differentials were performed on slides prepared in a Cytospin 3 (Shandon, Pittsburgh, PA, USA) centrifuged at $150 \mathrm{~g}$ for $2 \mathrm{~min}$ and stained with a modified Wright-Giemsa technique (Diff-Quik; Sysmex, Kobe, Japan). 


\section{Outcomes}

The primary outcome was the comparison of PCP-related mortality between the ILD group and non-ILD group. We defined the day when treatments of PCP were initiated as day 0 . The patients were followed for 90 days. PCP-related death was defined as death by respiratory failure after diagnosis of PCP. Patients withdrawn from the study due to other causes were censored. Secondary outcomes included the identification of prognostic factors of PCP and changes in serum markers ( $\beta$-D-glucan and Krebs von den Lungen-6 (KL-6)).

\section{Data collection and statistical analysis}

All clinical and laboratory data were collected from patients' medical records. Laboratory examination, HRCT and BAL were performed after the day of developing symptoms, defined as the onset of PCP. Continuous variables are expressed as median (interquartile range) and were compared using the Mann-Whitney U-test. For categorical variables, Fisher's exact test was performed. Survival analysis for all-cause mortality was performed using the Kaplan-Meier method with log-rank test. Cox proportional hazards regression models were used for univariate and multivariate analyses to determine the significant predictors of 90-day mortality, after adjusting for covariates of which the p-values were $<0.1$. The Wilcoxon signed rank test was used for comparisons of $\beta$-D-glucan and KL- 6 levels before and after PCP treatment, and between ILD and non-ILD groups. A p-value $<0.05$ was considered to indicate statistical significance. All statistical analyses were performed using the Statistical Package for the Social Sciences version 20.0 (SPSS Inc., Chicago, IL, USA).

\section{Results}

\section{Baseline characteristics}

A total of 139 patients were admitted with a diagnosis of PCP during the study period, of whom 89 patients met inclusion criteria (figure 1). 15 patients were excluded due to a HIV-positive diagnosis $(n=12)$ or lack of elevated serum $\beta$-D-glucan $(n=3)$. A total of 74 patients were included (ILD group: $n=32$; non-ILD group: $n=42$ ). No significant differences were observed between the two groups with regards to sociodemographic or baseline clinical variables (table 1). Diagnostic procedures for PCP were performed by GMS stain on BALF (25.7\%), PCR on BALF (51.4\%) and PCR on sputum (31.1\%), with no differences between the two groups. There were significant differences in median KL-6 ( $\mathrm{p}=0.001)$ and arterial oxygen tension $\left(P_{\mathrm{aO}_{2}}\right)$ /inspiratory oxygen fraction $\left(F_{\mathrm{IO}_{2}}\right)$ ratio $(\mathrm{p}=0.025)$. The median percentage of BALF neutrophils was significantly higher in the ILD group than in the non-ILD group $(p=0.012)$.

\section{Clinical diagnosis and HRCT pattern of ILD}

The clinical diagnosis and HRCT patterns of ILD are shown in table 2. Eight out of 17 patients with IIPs were diagnosed with IPF. 15 patients with non-IIPs were diagnosed with CTD-ILD ( $n=11)$, CHP ( $n=1)$, CEP ( $n=1)$, asbestosis $(\mathrm{n}=1)$ and radiation pneumonitis $(\mathrm{n}=1)$. 11 patients $(34.4 \%)$ showed a UIP pattern by HRCT.

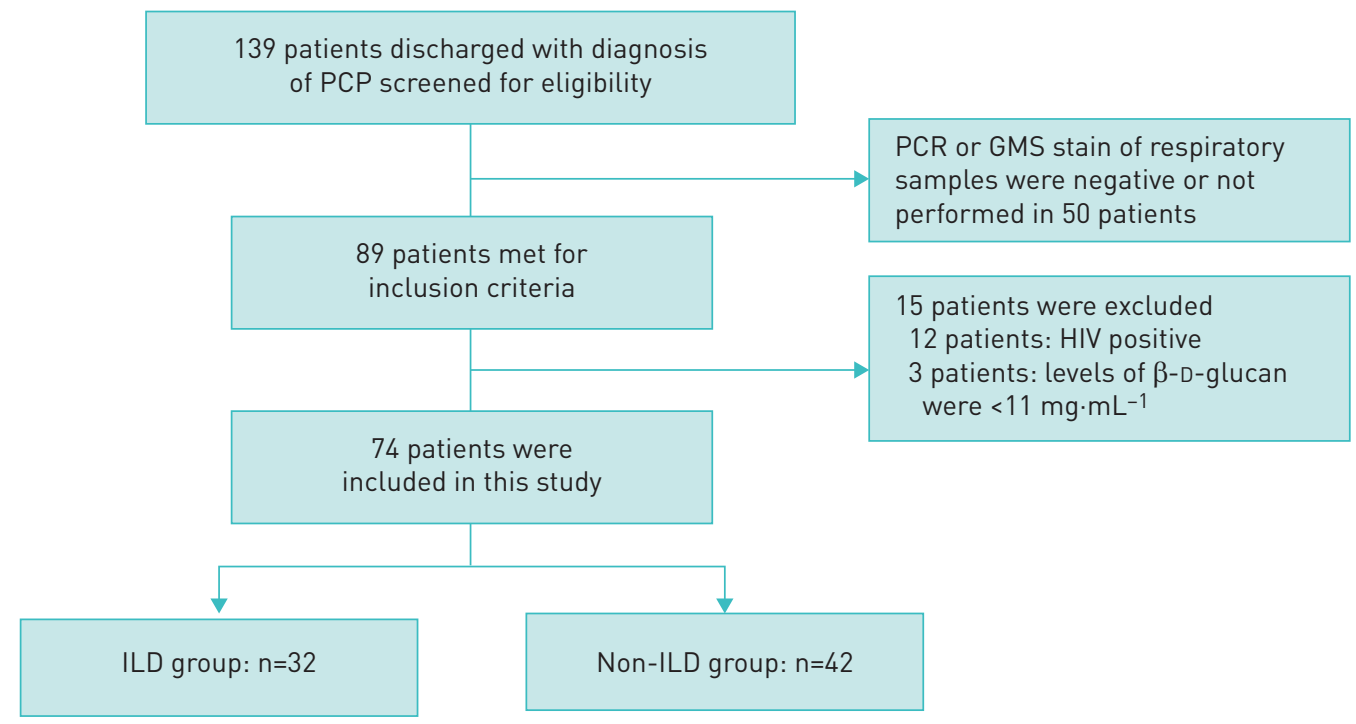

FIGURE 1 Inclusion and exclusion flow diagram. PCP: Pneumocystis pneumonia; GMS: Gomori methenamine silver; ILD: interstitial lung disease. 
TABLE 1 Patient characteristics

\begin{tabular}{|c|c|c|c|c|}
\hline & All & ILD group & non-ILD group & p-value \\
\hline Subjects & 74 & 32 & 42 & \\
\hline Age years & $73.5(66.0-80.0)$ & $74.0(68.5-80.8)$ & $68.0(66.5-73.0)$ & 0.125 \\
\hline Ever-smokers & $46(62.2 \%)$ & $20(62.5 \%)$ & $26(61.9 \%)$ & 1.000 \\
\hline BMI $\mathrm{kg} \cdot \mathrm{m}^{-2}$ & $21.8(19.5-24.0)$ & $22.4(19.1-24.0)$ & $21.0(19.8-24.0)$ & 0.291 \\
\hline Previous use of corticosteroids & $69(93.2 \%)$ & $31(96.9 \%)$ & $38(90.5 \%)$ & 0.381 \\
\hline \multicolumn{5}{|l|}{ Underlying diseases } \\
\hline IIPs & $9(12.2 \%)$ & $9(28.1 \%)$ & $0(0.0 \%)$ & $<0.001$ \\
\hline CTD & $24(32.4 \%)$ & $11(34.4 \%)$ & $13(31.0 \%)$ & 0.806 \\
\hline Solid tumour & $20(27.0 \%)$ & $5(15.6 \%)$ & $15(35.7 \%)$ & 0.067 \\
\hline Haematologic malignancy & $8(10.8 \%)$ & $2(6.3 \%)$ & $6(14.3 \%)$ & 0.453 \\
\hline Others & $13(17.6 \%)$ & $5(15.6 \%)$ & $8(19.0 \%)$ & 0.331 \\
\hline \multicolumn{5}{|l|}{ Laboratory findings } \\
\hline Lymphocyte count cells per $\mu \mathrm{L}$ & $695.0(346.0-1067.5)$ & $648.0(326.0-980.0)$ & $695.0(367.0-1100.0)$ & 0.688 \\
\hline Albumin $\mathrm{mg} \cdot \mathrm{dL}^{-1}$ & $2.7(2.3-3.1)$ & $2.6(2.4-2.9)$ & $2.8(2.2-3.4)$ & 0.271 \\
\hline $\mathrm{LDH} U \cdot \mathrm{L}^{-1}$ & 434.0 (295.0-540.5) & $434.0(298.0-544.0)$ & 453.0 (293.0-544.0) & 0.819 \\
\hline $\mathrm{KL}-6^{\#} \mathrm{U} \cdot \mathrm{mL}^{-1}$ & $754.0(476.0-1705.0)$ & 1170.0 (703.0-2271.8) & 585.5 (411.5-965.8) & 0.001 \\
\hline $\mathrm{CRP} \mathrm{mg} \cdot \mathrm{dL}^{-1}$ & $6.7(3.3-11.5)$ & $4.8(2.1-10.9)$ & $6.9(5.0-14.0)$ & 0.230 \\
\hline eGFR $\mathrm{mL} \cdot \mathrm{min}^{-1} \cdot 1.73 \mathrm{~m}^{-2}$ & $60.2(42.3-81.5)$ & $58.0(43.0-69.9)$ & $64.7(41.8-85.1)$ & 0.285 \\
\hline$\beta$-D-glucan pg.mL ${ }^{-1}$ & $87.0(66.0-80.0)$ & 78.4 (28.5-281.8) & $86.7(46.8-240.0)$ & 0.382 \\
\hline $\begin{array}{l}P_{\mathrm{aO}_{2}} / F_{\mathrm{IO}_{2}} \text { ratio } \\
\text { BALF findings }\end{array}$ & 248.0 (155.8-298.0) & $175.0(107.0-289.5)$ & 251.5 (180.0-301.0) & 0.025 \\
\hline Neutrophils & $12.2 \%(4.9-40.5 \%)$ & $22.6 \%(10.6-47.9 \%)$ & $6.6 \%(3.2-38.1 \%)$ & 0.012 \\
\hline Lymphocytes & $34.3 \%(11.3-55.3 \%)$ & $30.0 \%(9.5-48.0 \%)$ & $46.0 \%(8.75-64.9 \%)$ & 0.101 \\
\hline Eosinophils & $1.0 \%(0.0-2.3 \%)$ & $0.2 \%(0.0-2.0 \%)$ & $1.0 \%(0.0-3.0 \%)$ & 0.69 \\
\hline \multicolumn{5}{|c|}{$\begin{array}{l}\text { Data are presented as median linterquartile range) unless otherwise stated. ILD: interstitial lung disease; BMI: body mass index; } \\
\text { PCP: Pneumocystis pneumonia; IIP: idiopathic interstitial pneumonia; CTD: connective tissue disease; BALF: bronchoalveolar lavage fluid; } \\
\text { GMS: Gomori methenamine silver; LDH: lactate dehydrogenase; KL-6: Krebs von den Lungen-6; CRP: C-reactive protein; eGFR: estimated } \\
\text { glomerular filtration rate; } P_{\mathrm{aO}_{2}} \text { : arterial oxygen tension; } F_{\mathrm{IO}_{2}} \text { : inspiratory oxygen fraction. }{ }^{\#}: \mathrm{n}=69 ;{ }^{9}: \mathrm{n}=53 \text {. The p-values refer to comparisons } \\
\text { between the ILD and non-ILD groups. }\end{array}$} \\
\hline
\end{tabular}

\section{Treatment for PCP}

Therapeutic interventions performed during the study are shown in table S1. There were no significant differences in median days from onset to the initiation of therapy between the ILD group (8.0 days) and non-ILD group (10.3 days). Most patients were treated with trimethoprim/sulfamethoxazole (TMP/SMX) and adjunct corticosteroids. High-dose corticosteroid pulse therapy was applied to 15 patients (46.9\%) in the ILD group, which was significantly higher than in the non-ILD group $(9.5 \%, \mathrm{p}<0.001)$.

\section{Outcomes and prognostic factors of PCP}

The 90 -day mortality rate was $37.8 \%$ (28 out of 74 ) in all subjects and significantly higher in the ILD group $(62.5 \%, 20$ out of 32$)$ than in the non-ILD group $(19.0 \%$, eight out of $42 ; \mathrm{p}<0.001)$ (figure 2 ). After excluding the IIP patients in the ILD group, the 90-day mortality was also significantly higher in the ILD group than in the non-ILD group ( $53.3 \%$ versus $19.0 \%, \mathrm{p}=0.014$ ) (figure $\mathrm{S} 1$ ). In the ILD group, no significant differences in 90-day mortality were observed between patients with IIPs and non-IIPs (70.6\% versus $53.3 \%$ ) (figure S2a) or UIP pattern and non-UIP pattern (72.7\% versus $57.1 \%$ ) (figure S2b).

BALF neutrophilia in PCP patients was significantly related to survival rate. The subjects were categorised as a "higher" or "lower" group according to the median value of the percentage of BALF neutrophils. The median percentage of BALF neutrophils was $12.6 \%$ across all subjects (ILD group: $22.6 \%$; non-ILD group: $6.6 \%)$. BALF neutrophilia was significantly associated with the 90-day mortality rate when taking all subjects into account (higher group: 50.0\%; lower group: 17.4\%) $(\mathrm{p}=0.021)$ (figure 3a) and in the ILD 


\begin{tabular}{|c|c|}
\hline & Patients $\#$ \\
\hline \multicolumn{2}{|l|}{ Clinical diagnosis of ILD } \\
\hline IIPs & $17(53.1 \%)$ \\
\hline IPF & $8(25.0 \%)$ \\
\hline Non-IPF & $9(28.1 \%)$ \\
\hline Non-IIPs & $15(46.9 \%)$ \\
\hline CTD-ILD & $11(34.4 \%)$ \\
\hline $\mathrm{CHP}$ & $1(3.1 \%)$ \\
\hline CEP & $1(3.1 \%)$ \\
\hline Asbestosis & $1(3.1 \%)$ \\
\hline Radiation pneumonitis & $1(3.1 \%)$ \\
\hline \multicolumn{2}{|l|}{ HRCT patterns of ILD } \\
\hline UIP pattern & $11(34.4 \%)$ \\
\hline Non-UIP pattern & $21(65.6 \%)$ \\
\hline
\end{tabular}

group (higher group: 90.0\%; lower group: 50.0\%) ( $\mathrm{p}=0.026$ ) (figure $3 \mathrm{~b}$ ). No significant association was found for the non-ILD group (figure 3c).

We used a Cox hazards model for univariate and multivariate analyses to determine the significant predictors of 90-day mortality. In the univariate analysis, serum albumin, $\mathrm{LDH}, \mathrm{KL}-6, \mathrm{P}_{\mathrm{aO}} / F_{\mathrm{IO}_{2}}$ ratio, C-reactive protein, steroid pulse therapy and pre-existing ILD were covariates with a $\mathrm{p}$-value $<0.1$ (table 3 ). In the multivariate analysis, pre-existing ILD (hazard ratio (HR) 5.952, 95\% CI 1.900-18.649; $\mathrm{p}=0.002$ ) and the serum level of albumin (HR 0.312, 95\% CI 0.131-0.743; $\mathrm{p}=0.009$ ) were independent risk factors for 90-day mortality.

\section{Changes in $\beta$-D-glucan and KL-6 after PCP treatment}

We examined the changes in serum markers associated with PCP and ILD. The levels of $\beta$-D-glucan significantly decreased after the treatment of PCP in both the ILD group ( $p=0.006)$ (figure 4a) and non-ILD group ( $p=0.001$ ) (figure $4 \mathrm{~b}$ ). The serum levels of KL-6 significantly increased in the ILD group $(\mathrm{p}=0.001)$ (figure $4 \mathrm{c}$ ) and did not change in the non-ILD group $(\mathrm{p}=0.249)$ (figure $4 \mathrm{~d})$. In the ILD group, the 90-day mortality of 19 patients with increasing KL-6 after the treatment was significantly higher than that of five patients with decreasing KL-6 (78.9\% versus $0 \%, \mathrm{p}=0.019)$ (figure 5 ).

\section{Discussion}

We examined the impact of pre-existing ILD on the outcomes of non-HIV PCP. The mortality was significantly higher in the ILD group than in the non-ILD group. Multivariate analysis demonstrated that

FIGURE 2 Kaplan-Meier comparison of survival curves between the interstitial lung disease (ILD) and nonILD groups. The 90-day mortality was significantly higher in the ILD group than in the non-ILD group $162.5 \%$ versus 19.0\%). PCP: Pneumocystis pneumonia.

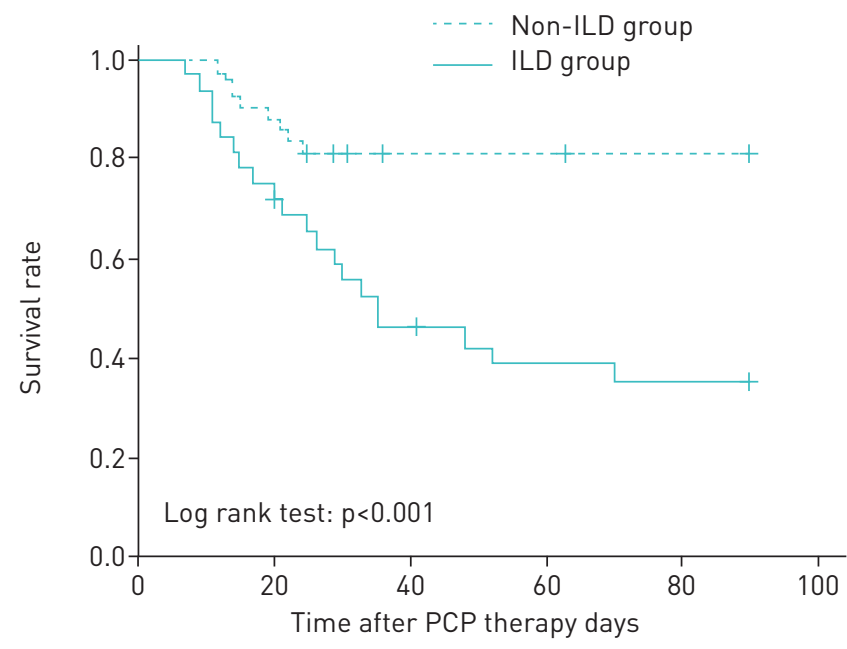



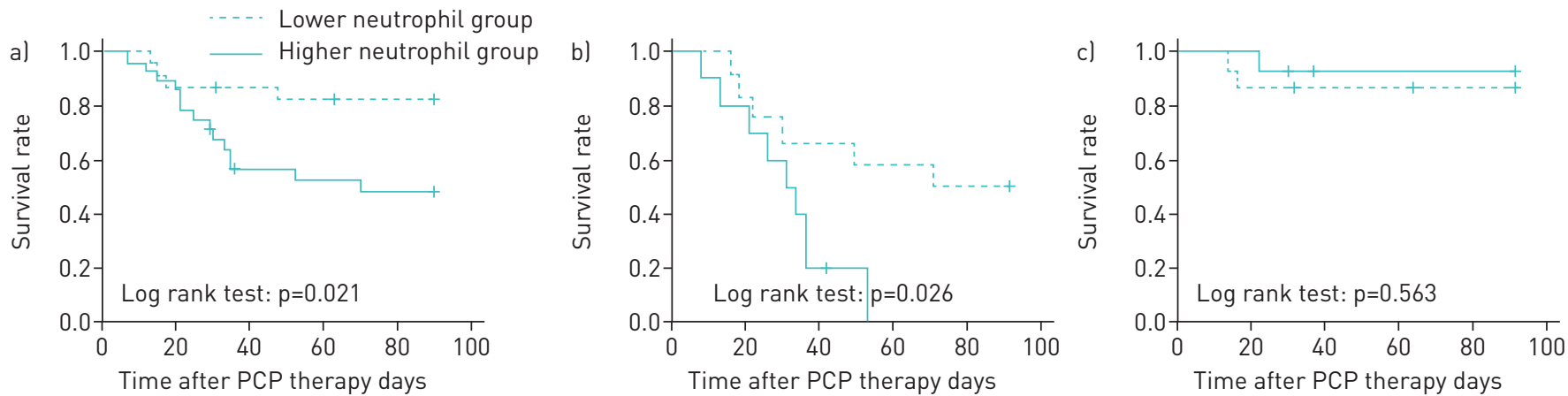

FIGURE 3 Kaplan-Meier comparison of survival curves between lower and higher percentage of bronchoalveolar lavage fluid (BALF) neutrophil groups. The patients were categorised into the "higher" or "lower" group according to the median percentage of BALF neutrophils. al With regards to all subjects as a whole, the 90-day survival was significantly worse in the higher group ( $\mathrm{n}=28$ ) than in the lower group ( $\mathrm{n}=23$ ) (90-day mortality rate in the higher group: $50.0 \%$; lower group: $17.4 \%$ ). b) In the interstitial lung disease (ILD) group, the 90 -day survival was significantly worse in the higher group ( $n=12$ ) than in the lower group ( $n=10$ ) (higher group: $90.0 \%$; lower group: 50.0\%). c) In the non-ILD group, the 90-day survival was not different between the higher group $(n=14)$ and the lower group $(n=15)$ (higher group: 13.3\%; lower group: $7.1 \%)$.

pre-existing ILD and serum levels of albumin were independent risk factors of 90-day mortality. Furthermore, this is the first study that has reported a significant increase in serum KL-6 levels after treatment of non-HIV PCP in patients with pre-existing ILD and a concurrent decrease in serum $\beta$-D-glucan levels.

Several risk factors, such as low serum albumin, high LDH and severe respiratory failure, have been shown to be associated with poorer prognosis in non-HIV patients with PCP [5-8]. In a recent review examining underlying diseases as risk factors for mortality, Liu et al. [6] reported a significant association for the presence of solid tumours. While coexisting pulmonary diseases have been reported as risk factors for developing PCP [23], the impact of pre-existing ILD on outcomes of PCP was unknown. A small study examining 20 non-HIV patients with PCP, including four patients with pre-existing ILD, reported a significant association between ILD and a high 90-day mortality [24]. AoKI et al. [25] investigated prognostic indicators in 25 PCP patients with CTD and concluded that pre-existing CTD-ILD was one of the prognostic factors leading to death. Nevertheless, there has been a lack of studies that have mainly focused on pre-existing ILD in non-HIV PCP. In our study, all patients with pre-existing ILD, which was regarded as an underlying disease of PCP or comorbid condition, were assigned to the ILD group. Consequently, a greater number of patients with pre-existing ILD were included in the present study than in previous studies, and we showed that pre-existing ILD was an important predictor for a poorer prognosis.

Severe PCP is characterised by intense neutrophilic pulmonary inflammation associated with diffuse alveolar damage (DAD), which is also observed as the typical pathological finding of ARDS and acute exacerbation (AE) of ILD, including AE-IPF [26-29]. According to our analysis of BALF cellular profiles, the percentage of BALF neutrophils was significantly higher in the ILD group than in the non-ILD group

\section{TABLE 3 Univariate and multivariate analyses of predictors of 90 -day mortality}

\begin{tabular}{|c|c|c|}
\hline Variables & HR $(95 \% \mathrm{CI})$ & p-value \\
\hline \multicolumn{3}{|l|}{ Univariate analysis } \\
\hline Pre-existing ILD & 4.003 (1.761-9.098) & 0.001 \\
\hline Steroid pulse therapy & $3.135(1.488-6.606)$ & 0.004 \\
\hline Albumin & $0.364(0.214-0.621)$ & $<0.001$ \\
\hline $\mathrm{KL}-6$ & $1.000(1.000-1.001)$ & 0.002 \\
\hline LDH & $1.002(1.000-1.003)$ & 0.074 \\
\hline CRP & $1.045(0.992-1.101)$ & 0.095 \\
\hline$P_{\mathrm{aO}_{2}} / F_{\mathrm{IO}_{2}}$ ratio & $0.991(0.987-0.996)$ & $<0.001$ \\
\hline \multicolumn{3}{|l|}{ Multivariate analysis } \\
\hline Pre-existing ILD & $5.952(1.900-18.649)$ & 0.002 \\
\hline Albumin & $0.312(0.131-0.743)$ & 0.009 \\
\hline
\end{tabular}



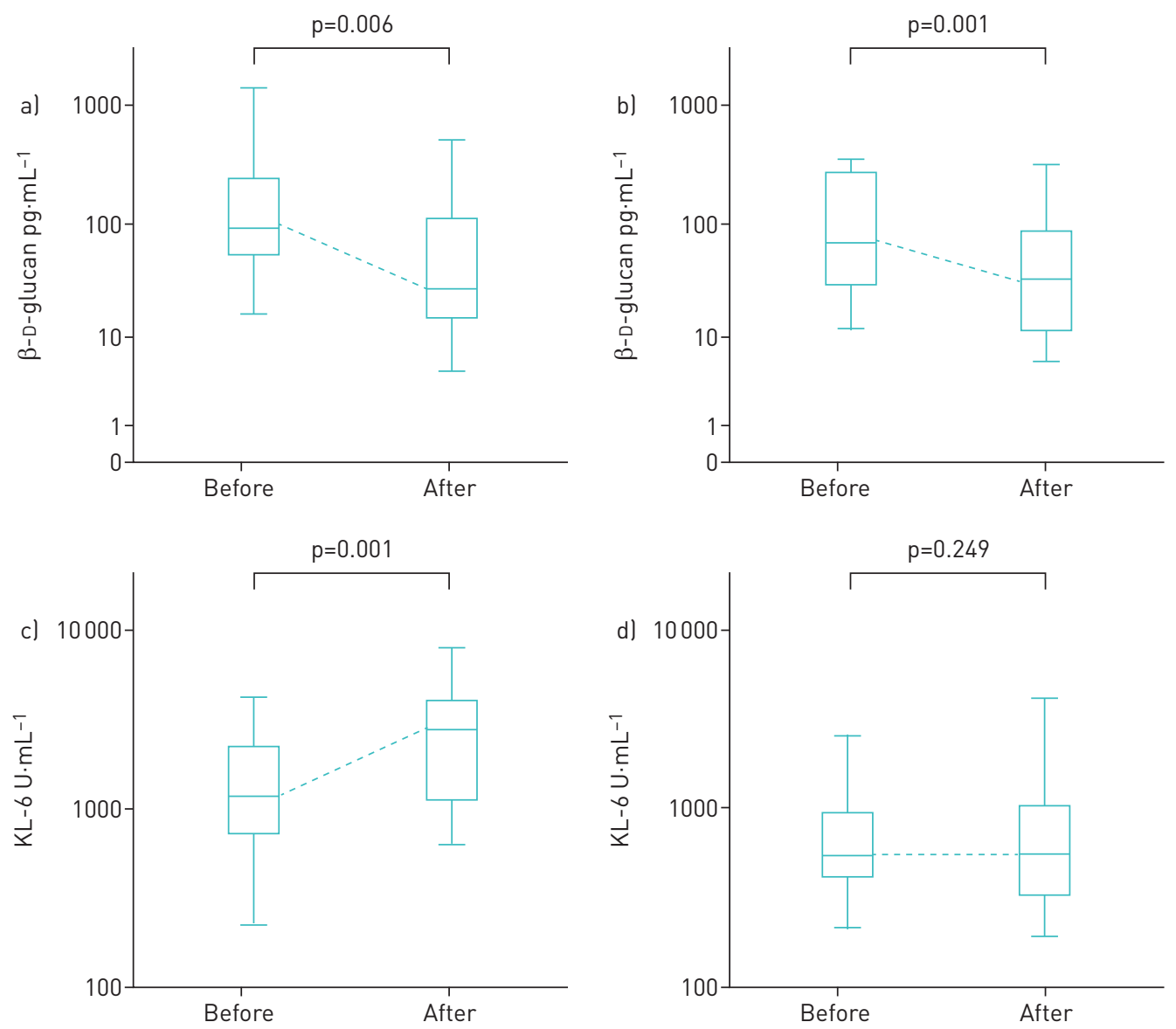

FIGURE 4 Comparisons of serum levels of $\beta$-D-glucan and Krebs von den Lungen- 6 (KL-6) before and after Pneumocystis pneumonia treatment. The levels of $\beta$-D-glucan significantly decreased in both groups: a) median value of the interstitial lung disease (ILD) group from 78.5 to $38.5 \mathrm{pg} \cdot \mathrm{mL}^{-1}, \mathrm{p}=0.006$; b) non-ILD group from 95.0 to $\left.27.0 \mathrm{pg} \cdot \mathrm{mL}^{-1}, \mathrm{p}=0.001\right)$. c) The level of $\mathrm{KL}-6$ significantly increased in the ILD group (median value from 1505.0 to $2730.0 \mathrm{U} \cdot \mathrm{mL}^{-1}, \mathrm{p}=0.001$ ) and d) did not change significantly in the non-ILD group (median value from 659.5 to $550.0 \mathrm{U} \cdot \mathrm{mL}^{-1}, \mathrm{p}=0.249$ ).

and this factor was significantly associated with a poorer prognosis. These results suggest that in patients with PCP, pre-existing ILD may increase susceptibility to the development of DAD, namely AE-ILD, leading to refractory respiratory failure. However, in the non-ILD group, the degree of BALF neutrophilia was not associated with the survival rate. This contrasts with several studies that have reported that BALF neutrophilia may be a significant prognostic factor associated with fatality in patients with non-HIV PCP

FIGURE 5 Kaplan-Meier comparison of survival curves in the interstitial lung disease patients divided by increasing or decreasing Krebs von den Lungen-6 (KL-6) levels after Pneumocystis pneumonia (PCP) treatment. The 90-day mortality of the patients with increasing KL-6 after the treatment was significantly higher than those with decreasing $\mathrm{KL}-6$ lincreasing $K L-6, \quad n=19: 78.9 \%$; decreasing $K L-6, n=5$ : $0 \%$ ).

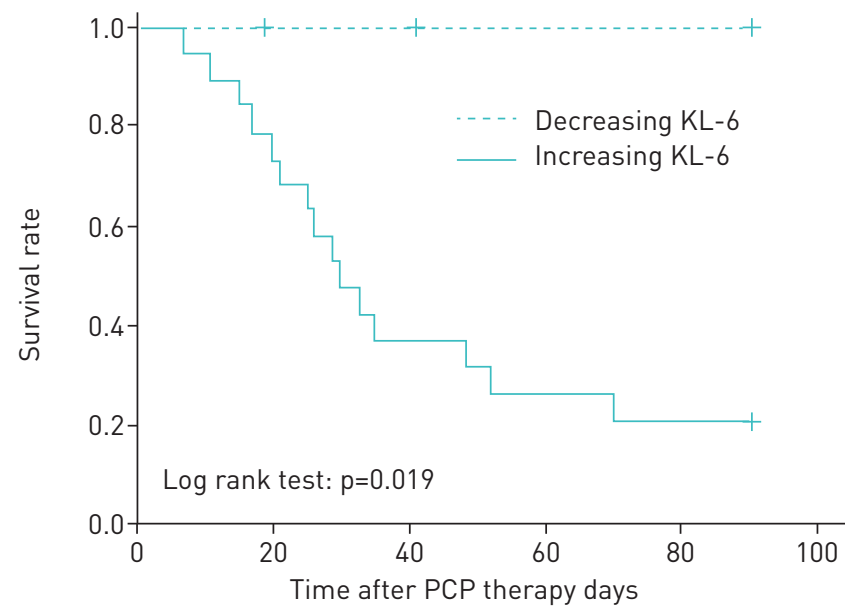


in the absence of pre-existing ILD [30, 31]. While the reasons for this discrepancy are unclear, previous studies have reported that the extent of lung damage in neutrophil-impaired knockout mice with PCP were unexpectedly the same as in wild-type mice, and $\mathrm{CD}^{+} \mathrm{T}$-cell-mediated responses might be more directly responsible for lung injury than neutrophil-mediated inflammation [32, 33]. As only limited previous data on the prognostic utility of BALF cellular analysis in non-HIV PCP are available, further research is required to elucidate the exact role of neutrophils in the pathogenesis of PCP.

The serum level of KL-6 is known to be useful for not only detecting the presence of ILD and evaluating disease activity, but also for the diagnosis of PCP [34, 35]. In the present study, serum levels of KL-6 were elevated at the time of PCP diagnosis, regardless of the presence or absence of pre-existing ILD, and the elevation of KL-6 was higher in the ILD group than in the non-ILD group. While KL-6 levels were significantly increased in the ILD group and did not change in the non-ILD group after PCP treatment, levels of $\beta$-D-glucan were significantly reduced in both groups. These paradoxical changes in KL- 6 and $\beta$-D-glucan levels in the ILD group might be attributed to the progressive and refractory characteristics of pre-existing ILD after treatment for PCP. Furthermore, in the ILD group, the mortality of patients with increasing KL-6 was significantly higher compared to patients with decreasing KL-6. Үокоунма et al. [36] also reported that an increase in KL-6 after treatment predicted poorer outcomes in cases of rapidly progressive IPF, including AE-IPF, suggesting that the monitoring of KL-6 may be useful for the management of active IPF. The utility of KL-6 measurement in PCP patients, with or without ILD, needs to be addressed in future studies.

In our study, the prognosis of the ILD group was very poor despite sufficient treatment. Notably, no patients in the ILD group had received prophylaxis for PCP with TMP/SMX. In a meta-analysis investigating the efficacy of prophylaxis for non-HIV immunocompromised patients, the incidence of developing PCP was $6.2 \%$ in the control group versus $0.9 \%$ in the prophylaxis group [37]. Although some attending physicians in our study did not perform or discontinued prophylaxis due to renal or hepatic disorders and side-effects such as skin rash and leukopenia (data not shown), we suggest that it is prudent to provide prophylaxis for PCP whenever possible in patients with pre-existing ILD under immunosuppression.

This study has several limitations. First, this was a retrospective study employing a small sample, and did not randomise patients to treatment and control groups. Second, the underlying comorbid conditions were heterogeneous and could have affected the outcomes of PCP treatment. Third, there was insufficient previous laboratory data regarding KL- 6 and $\beta$-D-glucan measurements before the onset of PCP, and newly developed GGO could be observed in both PCP and AE-ILD. Furthermore, the diagnosis of PCP was based on PCR results in three-quarters of all patients, and false positive results may have occurred due to colonisation by $P$. jirovecii as opposed to true infection. Therefore, we could not completely distinguish PCP from AE of pre-existing ILD. Nevertheless, the levels of $\beta$-D-glucan were significantly elevated at diagnosis in all patients and reduced after PCP therapy in most patients, suggesting a more accurate diagnosis of PCP than in prior studies [38].

In conclusion, our study indicates that pre-existing ILD is associated with a poorer prognosis in patients with non-HIV PCP. Therefore, prophylaxis for PCP should be considered, especially in patients with pre-existing ILD under immunosuppression.

Acknowledgements: We would like to acknowledge all the participants. The authors would like to thank Editage (https:// www.editage.jp) for the professional English language review.

Conflict of interest: None declared.

\section{References}

1 Kelley CF, Checkley W, Mannino DM, et al. Trends in hospitalizations for AIDS-associated Pneumocystis jirovecii pneumonia in the United States (1986 to 2005). Chest 2009; 136: 190-197.

2 Buchacz K, Baker RK, Palella FJ Jr, et al. AIDS-defining opportunistic illnesses in US patients, 1994-2007: a cohort study. AIDS 2010; 24: 1549-1559.

3 Bienvenu AL, Traore K, Plekhanova I, et al. Pneumocystis pneumonia suspected cases in 604 non-HIV and HIV patients. Int J Infect Dis 2016; 46: 11-17.

4 Enomoto T, Azuma A, Kohno A, et al. Differences in the clinical characteristics of Pneumocystis jirovecii pneumonia in immunocompromised patients with and without HIV infection. Respirology 2010; 15: 126-131.

5 Kim SJ, Lee J, Cho YJ, et al. Prognostic factors of Pneumocystis jirovecii pneumonia in patients without HIV infection. J Infect 2014; 69: 88-95.

6 Liu Y, Su L, Jiang SJ, et al. Risk factors for mortality from Pneumocystis carinii pneumonia (PCP) in non-HIV patients: a meta-analysis. Oncotarget 2017; 8: 59729-59739.

7 Hardak E, Neuberger A, Yigla M, et al. Outcome of Pneumocystis jirovecii pneumonia diagnosed by polymerase chain reaction in patients without human immunodeficiency virus infection. Respirology 2012; 17: 681-686.

8 Schmidt JJ, Lueck C, Ziesing S, et al. Clinical course, treatment and outcome of Pneumocystis pneumonia in immunocompromised adults: a retrospective analysis over 17 years. Crit Care 2018; 22: 307. 
9 Shimizu Y, Sunaga N, Dobashi K, et al. Serum markers in interstitial pneumonia with and without Pneumocystis jirovecii colonization: a prospective study. BMC Infect Dis 2009; 22: 47.

10 Azadeh N, Limper AH, Carmona EM, et al. The role of infection in interstitial lung diseases: a review. Chest 2017; 152: 842-852.

11 Moua T, Westerly BD, Dulohery MM, et al. Patients with fibrotic interstitial lung disease hospitalised for acute respiratory worsening: a large cohort analysis. Chest 2016; 149: 1205-1214.

12 Gannon WD, Lederer DJ, Biscotti M, et al. Outcomes and mortality prediction model of critically ill adults with acute respiratory failure and interstitial lung disease. Chest 2018; 153: 1387-1395.

13 Wang CY, Calfee CS, Paul DW, et al. One-year mortality and predictors of death among hospital survivors of acute respiratory distress syndrome. Intensive Care Med 2014; 40: 388-396.

14 Bellani G, Laffey JG, Pham T, et al. Epidemiology, patterns of care, and mortality for patients with acute respiratory distress syndrome in intensive care units in 50 countries. JAMA 2016; 315: 788-800.

15 Vogel MN, Vatlach M, Weissgerber P, et al. HRCT-features of Pneumocystis jirovecii pneumonia and their evolution before and after treatment in non-HIV immunocompromised patients. Eur J Radiol 2012; 81: $1315-1320$.

16 Travis WD, Costabel U, Hansell DM, et al. An official American Thoracic Society/European Respiratory Society statement: update of the international multidisciplinary classification of the idiopathic interstitial pneumonias. $A m$ J Respir Crit Care Med 2013; 188: 733-748.

17 Vij R, Strek ME. Diagnosis and treatment of connective tissue disease-associated interstitial lung disease. Chest 2013; 143: 814-824.

18 Vasakova M, Morell F, Walsh S, et al. Hypersensitivity pneumonitis: perspectives in diagnosis and management. Am J Respir Crit Care Med 2017; 196: 680-689.

19 Valent P, Klion AD, Horny HP, et al. Contemporary consensus proposal on criteria and classification of eosinophilic disorders and related syndromes. J Allergy Clin Immunol 2012; 130: 607-612.

20 Ghio AJ, Roggli VL. Diagnosis and initial management of nonmalignant diseases related to asbestos. Am J Respir Crit Care Med 2005; 171: 527-530.

21 Movsas B, Raffin TA, Epstein AH, et al. Pulmonary radiation injury. Chest 1997; 111: 1061-1076.

22 Raghu G, Remy-Jardin M, Myers JL, et al. Diagnosis of idiopathic pulmonary fibrosis. An Official ATS/ERS/JRS/ ALAT clinical practice guideline. Am J Respir Crit Care Med 2018; 198: e44-e68.

23 Harigai M, Koike R, Miyasaka N, et al. Pneumocystis pneumonia associated with infliximab in Japan. $N$ Engl Med 2007; 357: 1874-1876.

24 Kotani T, Katayama S, Miyazaki Y, et al. Risk factors for the mortality of Pneumocystis jirovecii pneumonia in non-HIV patients who required mechanical ventilation: a retrospective case series study. Biomed Res Int 2017; 2017: 7452604

25 Aoki Y, Iwamoto M, Kamata Y, et al. Prognostic indicators related to death in patients with Pneumocystis pneumonia associated with collagen vascular diseases. Rheumatol Int 2009; 29: 1327-1330.

26 Wright TW, Gigliotti F, Finkelstein JN, et al. Immune-mediated inflammation directly impairs pulmonary function, contributing to the pathogenesis of Pneumocystis carinii pneumonia. J Clin Invest 1999; 104: 1307-1317.

27 Thomas CF Jr, Limper AH. Current insights into the biology and pathogenesis of Pneumocystis pneumonia. Nat Rev Microbiol 2007; 5: 298-308.

28 Collard HR, Ryerson CJ, Corte TJ, et al. Acute exacerbation of idiopathic pulmonary fibrosis. An international working group report. Am J Respir Crit Care Med 2016; 194: 265-275.

29 Kolb M, Bondue B, Pesci A, et al. Acute exacerbations of progressive-fibrosing interstitial lung diseases. Eur Respir Rev 2018; 27: 180071.

30 Zahar JR, Robin M, Azoulay E, et al. Pneumocystis carinii pneumonia in critically ill patients with malignancy: a descriptive study. Clin Infect Dis 2002; 35: 929-934.

31 Tamai K, Tachikawa R, Tomii K, et al. Prognostic value of bronchoalveolar lavage in patients with non-HIV Pneumocystis pneumonia. Intern Med 2014; 53: 1113-1117.

32 Swain SD, Wright TW, Degel PM, et al. Neither neutrophils nor reactive oxygen species contribute to tissue damage during Pneumocystis pneumonia in mice. Infect Immun 2004; 72: 5722-5732.

33 Swain SD, Meissner NN, Harmsen AG. CD8 T cells modulate CD4 T-cell and eosinophil-mediated pulmonary pathology in pneumocystis pneumonia in B-cell-deficient mice. Am J Pathol 2006; 168: 466-475.

34 Tasaka S, Hasegawa N, Kobayashi S, et al. Serum indicators for the diagnosis of Pneumocystis pneumonia. Chest 2007; 131: 1173-1180

35 Ishikawa N, Hattori N, Yokoyama A, et al. Utility of KL-6/MUC1 in the clinical management of interstitial lung diseases. Respir Investig 2012; 50: 3-13.

36 Yokoyama A, Kohno N, Hamada H, et al. Circulating KL-6 predicts the outcome of rapidly progressive idiopathic pulmonary fibrosis. Am J Respir Crit Care Med 1998; 158: 1680-1684.

37 Stern A, Green H, Paul M, et al. Prophylaxis for Pneumocystis pneumonia (PCP) in non-HIV immunocompromised patients. Cochrane Database Syst Rev 2014; 1: CD005590.

38 Matsumura Y, Ito Y, Iinuma Y, et al. Quantitative real-time PCR and the $(1 \rightarrow 3)-\beta$-D-glucan assay for differentiation between Pneumocystis jirovecii pneumonia and colonization. Clin Microbiol Infect 2012; 18: 591-597. 